\title{
Relationship between the correction angle in medial open wedge high tibial osteotomy and the postoperative state of patellofemoral cartilage
}

\section{Chong-Hyuk Choi}

Arthroscopy and Joint Research Institute, Severance Hospital, Yonsei University College of Medicine Min Jung

Arthroscopy and Joint Research Institute, Severance Hospital, Yonsei University College of Medicine

\section{Sung-Hwan Kim}

Arthroscopy and Joint Research Institute, Severance Hospital, Yonsei University College of Medicine

\section{Sang-Hee Kim}

Arthroscopy an Joint Research Institute, Severance Hospital, Yonsei University College of Medicine

Tae-Hwan Yoon ( $\square$ uvsoul@yuhs.ac)

Yonsei University Health System https://orcid.org/0000-0002-2859-5240

Research article

Keywords: medial open wedge high tibial osteotomy, osteoarthritis, patellofemoral arthritis, correction angle

Posted Date: January 15th, 2020

DOI: https://doi.org/10.21203/rs.2.20950/v1

License: (c) (1) This work is licensed under a Creative Commons Attribution 4.0 International License. Read Full License 


\section{Abstract}

Background: To investigate clinical and radiologic effects of medial open wedge high tibial osteotomy (MOWHTO) on the patellofemoral joint in relation to the correction angle by comparing changes in cartilage from before to after the surgery.

Methods: A total of 124 MOWHTO cases were divided into the three groups of small, moderate, and large, depending on the correction angle. Clinical and radiologic outcomes were compared at the mean followup time of 38.8 months. Postoperative cartilage changes were assessed during implant removal completed at two years after MOWHTO.

Results: There was no significant difference evident in most clinical outcomes measured, including Lysholm score and the Knee Injury and Osteoarthritis Outcome score subscales, with the exception of postoperative Shelbourne and Trumper score between the groups $(P<0.001)$. International Knee Documentation Committee scale value on radiologic evaluation did not significantly differ among the groups. A progression of cartilage degeneration was noted in $40.3 \%$ of femoral trochleas and $22.6 \%$ of patellas. Cartilage degeneration was significantly progressed in the large correction angle group versus in the other two groups. Regression analysis showed that the correction angle had a significant effect on cartilage deterioration (Trochela, $\mathrm{P}=0.009$; Patella, $\mathrm{P}=0.034$ ).

Conclusion: Patellofemoral joint was more adversely affected by MOWHTO in conjunction with the requirement of a larger correction angle. Thus, cases necessitating considerable correction should be forewarned of patellofemoral joint symptoms within a relatively short time after the procedure.

Keywords: medial open wedge high tibial osteotomy, osteoarthritis, patellofemoral arthritis, correction angle

\section{Background}

Medial open wedge high tibial osteotomy (MOWHTO) was first introduced by Debeyre, in 1951.[1] Since then, correction and fixation techniques of the osteotomy has been in constant development over time. Currently, it is considered one of the surgical options to treat medial compartment osteoarthritis that results in a good clinical outcome.[2-5] MOWHTO employs the principle of decompression in the medial compartment of the knee joint by shifting the mechanical axis to the lateral compartment.[6-8] Some of previous studies have demonstrated the effectiveness of MOWHTO in preventing further progression of arthritis at medial compartment and additionally in achieving substantial fibrocartilage coverage on the cartilage defect area.[9-11]

Because the main target of the MOWHTO is coronal realignment of the low extremity and decompression of the medial compartment, most of previous studies regarding the MOWHTO have focused on its therapeutic effect on the medial compartment. However, these studies have had a tendency to overlook the influence of realignment on another important part of the knee joint, the patellofemoral joint. 
It is only recently that study on the postoperative effect of MOWHTO on the patellofemoral joint has begun to attract attention. Several biomechanical studies have shown that distalization and lateralization of the tibial tuberosity caused by MOWHTO lead to loss in patellar height.[12-15] Loss in patellar height leads to an increase in patellofemoral contact pressure, which in turn may cause a higher incidence of arthritic change of patellofemoral joint and anterior knee pain.[15, 16] On the basis of this biomechanical finding, a few researchers have performed clinical studies with second-look arthroscopy on the knee treated with MOWHTO to examine the postoperative state of patellofemoral joint.[17-19] Although these clinical studies have agreed that the MOWHTO cause some degenerative progression in the patellofemoral joint of many patients, the reported postoperative functional assessment showed inconsistent results between the studies, making it difficult to reach a definite conclusion on the clinical effect of MOWHTO on the patellofemoral joint. Furthermore, there is a lack of study on the influential factors affecting the postoperative state of patellofemoral joint in MOWHTO. Since various clinical outcomes have been derived from the previous studies on the relationship between MOWHTO and postoperative change of patellofemoral joint,[17-19] the present study investigated the factors affecting the postoperative state of patellofemoral joint in MOWHTO focusing on the effect of the correction angle on the patellofemoral joint. The present study assessed clinical outcomes and arthroscopic findings of the patellofemoral joint in accordance with the correction angle after MOWHTO. The purpose of this study was to compare changes in cartilage states of the patellofemoral joint both preoperatively and postoperatively, to evaluate the clinical and radiologic outcomes, and consequently to determine how correction angle affects the postoperative state of patellofemoral joint after MOWHTO. It was hypothesized that a larger correction angle by MOWHTO would result in the more progression of cartilage degeneration in the patellofemoral joint and poorer clinical outcomes.

\section{Methods}

The current study retrospectively reviewed consecutive 165 knees (152 patients) who underwent MOWHTO with the TomoFix ${ }^{\circledR}$ implant (DePuy Synthes, Solothurn, Switzerland) between March 2008 and May 2013. Inclusion criteria were (1) symptomatic osteoarthritis at medial compartment with KellgrenLawrence grade of III or IV which did not respond to non-operative treatments for at least 3 months; (2) varus deformity of $>5^{\circ}$; (3) preserved articular cartilage of the lateral joint compartment with International Cartilage Repair Society (ICRS) grade of I or II, without tear of lateral meniscus;[20] (4) available for minimum two-year clinical and radiological follow-up after surgery. Exclusion criteria were (1) patients who were not able to undergo second-look arthroscopy; (2) complications including nonunion, malunion, and hardware failure; (3) a history of a previous surgery to the affected knee; (4) patient who had operation on both knees; (5) patients who had undergone a concurrent cartilage restoration procedure during MOWHTO; and (6) knee joint instability of the affected joint caused by any coexisting ligament problem. Finally, 124 knees who met the above criteria were included in this study. Our institutional review board approved this study.

Included patients were divided into three groups depending on the correction angle. The medial proximal tibial angle (MPTA) was measured on knee standing anteroposterior (AP) view before initial MOWHTO 
and just prior to the second surgery of implant removal. The MTPA was defined as the angle obtained from an intersection between the articular surface of the proximal tibia and the tibial mechanical axis (Fig. 1). The correction angle was defined by subtracting MPTA measured before the second surgery from the initial MPTA.[21] The calculated correction angles were divided into quartiles and the patients were subsequently divided into three groups on the basis of the interquartile range of the correction angles. The first group consisted of patients who were included in the lowest quartile $\left(<7.8^{\circ}\right)$; these individuals were defined as the small correction angle group (Group $S=31$ patients). The second group of patients, who had correction angles in the middle two quartiles $\left(7.8^{\circ}\right.$ to $\left.11.6^{\circ}\right)$, were named as the moderate correction angle group (Group $\mathrm{M}=62$ patients). Patients with a large correction angle, who were included in upper quartile $\left(>11.6^{\circ}\right)$, were classified as the large correction angle group (Group $L=31$ patients).

\section{Surgical Procedure}

A single surgeon performed all operations. AP full-length weight-bearing radiograph was taken for preoperative planning, and the target angle of valgus correction was measured with use of Miniaci's method.[22] The realigned weight-bearing line was planned to pass through the Fujisawa point $(62.5 \%$ from the medial edge of the tibial plateau).[23] Arthroscopic examination was initially done to assess the medial, lateral, and patellofemoral joint. And then, a skin incision was made obliquely on the medial proximal tibia and distal insertion of the superficial medial collateral ligament was stripped off the bone. Osteotomy site was usually determined near the upper border of the pes anserius. Under the guide of an image intensifier, two Kirschner wires were inserted toward just proximal to the tibiofibular joint. Along the Kirschner wires osteotomy was performed. The lateral-most $10 \mathrm{~mm}$ of the proximal tibia was preserved without damage in order to be used as a hinge during correction. A separate ascending osteotomy for the biplanar cut was performed about $1 \mathrm{~cm}$ to $1.5 \mathrm{~cm}$ posterior to the tibial tuberosity, and it was cut at an angle of $100^{\circ}$ to $110^{\circ}$ from the direction of initial osteotomy. Using four chisels, the osteotomy site was gradually opened up. To open and measure the gap of the osteotomy, the TomoFix bone spreader was inserted into the osteotomy gap. The opening process was performed such that the anterior gap of the opening was about two-thirds the size of the posterior opening to maintain the tibial slope.[24] Fixation of the osteotomy was done with the TomoFix ${ }^{\circledR}$ plate (DePuy Synthes, Solothurn, Switzerland) and locking screws.

\section{Postoperative Rehabilitation and Implant Removal}

A splint was applied for initial two weeks, and then knee brace was worn for next four weeks, during which time range of motion (ROM) of the knee joint was allowed within the tolerable range. Partial weight-bearing was initiated beginning at two weeks after the operation. The brace was discarded, and full-weight bearing was allowed at six weeks. Implant removal was performed at two year postoperatively with confirmation of bone union under simple radiography. Second-look arthroscopy to evaluate the cartilage state was also performed during the operation of removal.

\section{Radiologic Evaluation}


For radiological assessments, standing true anteroposterior full-length lower extremity radiograph, anteroposterior and lateral view of the both knee weight bearing radiographs, and $45^{\circ}$ Merchant view were taken. In addition to MPTA, the weight bearing line ratio, hip-knee-ankle angle, modified BlackburnPeel ratio, and the International Knee Documentation Committee (IKDC) radiographic scale value were evaluated by two orthopedic surgeons. Their evaluations were performed independently of each other, and were completed preoperatively and at the last follow-up for all patients. For both the preoperative and last evaluations, each surgeon measured these parameters twice in all knees, with an interval of two weeks between the measurements. The average of these two measurements was used for final analysis.

The weight bearing line ratio was obtained by first drawing a weight bearing line from the center of the femoral head to the midpoint of the talar superior articular surface; subsequently, the horizontal distance from the medial edge of the tibia plateau to the tibial intersection of the weight bearing line was recorded. With the medial edge at $0 \%$ and the lateral edge at $100 \%$, the weight bearing line ratio was set as a ratio of the horizontal distance from the medial edge of the tibia divided by the whole width of the tibia (Fig. 2A, B). The hip-knee-ankle angle was measured by drawing a line from the femoral head center to the tibial spine midpoint and another line extended from this midpoint to the talar surface center of the ankle joint. The angle made by the intersection of these two lines was determined as the hip-knee-ankle angle (Fig. 2C). The modified Blackburn-Peel ratio was assessed with standing lateral view of $30^{\circ}$ knee flexion. The ratio was set as the perpendicular distance between the tibial articular surface and the inferior end point of the patellar articular surface divided by the entire length of the articular surface of patella (Fig. 2-D). The radiologic evaluation of the patellofemoral joint on osteoarthritis was performed with the IKDC radiographic assessment scale on $45^{\circ}$ Merchant view, and the scale was categorized as follows: $A=$ normal; $\mathrm{B}=$ nearly normal, showing $>4 \mathrm{~mm}$ of a joint space with evidence of minimal changes in the joint including early osteophytes, slight sclerosis, or flattening of the femoral condyle; $\mathrm{C}=$ abnormal, showing a joint space of between $2 \mathrm{~mm}$ and $4 \mathrm{~mm}$ in height or up to $50 \%$ of joint-space narrowing; $\mathrm{D}=$ severely abnormal, showing a joint space with less than $2 \mathrm{~mm}$ or less than $50 \%$ of joint space remaining.[25] All radiologic evaluations were performed with the picture archiving and communication system (PACS) (Centricity ${ }^{\circledR}$ PACS viewer; GE Healthcare Co., Piscataway, NJ, USA).

\section{Functional Evaluation}

Functional evaluation of the patients was performed with Lysholm score[26] and Knee Injury and Osteoarthritis Outcome score (KOOS), including its subscales of KOOS Pain, KOOS Symptoms, KOOS Activities of Daily Living, KOOS Sports/Rec, and KOOS Quality of Life,[27] while clinical outcome of the patellofemoral joint was measured with the Shelbourne and Trumper questionnaire.[28] An independent examiner who was blinded to group assignment assessed the all of the above-mentioned clinical evaluations that were performed both preoperatively and at the time of the last follow-up.

\section{Arthroscopic Examination of the Articular Cartilage Status}

Changes in patellofemoral articular cartilage between initial and second-look arthroscopy were compared. Grades of the cartilage status were evaluated for both the patellar and femoral trochlea 
surfaces with ICRS grade, (Fig. 3-A, B). Grading results were first determined by a single operator, with the finding subsequently needing to be concurred with by two trained fellows. When the cartilage showed improvement with partial or full recovery at the defect area or if there was no interval change between the findings of initial and second-look arthroscopy, it was categorized into the non-progressed group. Cases with any evidence of progressive change in either the patellar or femoral trochlear cartilage were categorized in the progressed group.

\section{Statistical Analysis}

One-way analysis of variance (ANOVA) was used for normally distributed continuous variables. If the result of the ANOVA test had statistical significance, subsequent post-hoc analysis (Bonferroni's test) was performed. The Kruskal-Wallis test was first applied for non-normal distribution and the Mann-Whitney $\mathrm{U}$ test was then carried out as the post-hoc analysis for any result that had statistical significance. The chi-square test or Fisher's exact test was employed to compare categorical variables. Logistic regression analysis was used to determine any effect of independent variables such as age, sex, body mass index, preoperative hip-knee-ankle angle, postoperative modified Blackburn-Peel ratio, and correction angle on the cartilage status progression of patellofemoral joint. The intraclass correlation coefficient (ICC) was calculated to evaluate both intraobserver and interobserver reliabilities of the measured values. Statistical analysis was done using SPSS software (version 23.0; IBM Co., Armonk, NY, USA). A p-value of less than 0.05 was set to be statistically significant. The statistical power was calculated with the use of PASS software (version 11, NCSS, LLC, Kaysville, Utah, USA).

\section{Results}

The mean correction angles were $6.9^{\circ} \pm 0.6^{\circ}, 10.0^{\circ} \pm 1.1^{\circ}$, and $13.2^{\circ} \pm 1.0^{\circ}$ for Groups $S, M$, and $L$, respectively. The mean ages at the time of surgery were $56.6 \pm 2.4$ years in Group S, $55.6 \pm 3.4$ years in Group M, and $56.3 \pm 2.6$ years in Group L. There were eight males and 23 females in Group S, 14 males and 48 females in Group M, and nine males and 22 females in Group L. The mean follow-up period was $38.8 \pm 4.7$ months. The three groups did not differ significantly in terms of age, sex, affected side, body mass index, the mean follow-up period, and the elapse time from the initial surgery to the implant removal (Table 1). Preoperative functional evaluations such as Lysholm knee score, KOOS Pain, KOOS Symptoms, KOOS Activities of Daily Living, KOOS Sport/Rec, and KOOS Quality of Life did not differ between groups. Radiological evaluation regarding the MPTA, modified Blackburn-Peel ratio, and IKDC radiographic scale did not show significant difference between the groups. The only statistically significant difference was found in the preoperative hip-knee-ankle angle, in which Groups $S, M$, and $L$ had mean angles of $-6.9^{\circ} \pm 2.5^{\circ},-8.2^{\circ} \pm 2.1^{\circ}$, and $-10.0^{\circ} \pm 3.4^{\circ}$, respectively $(p<0.001)$ (Table 2$)$. The preoperative proportion of ICRS grade of the femoral trochlea and patella did not differ between the groups. 
Table 1

Preoperative demographic characteristics

\begin{tabular}{|c|c|c|c|c|}
\hline & $\begin{array}{l}\text { Group S } \\
(n=31)\end{array}$ & $\begin{array}{l}\text { Group } \\
M \\
(n=62)\end{array}$ & $\begin{array}{l}\text { Group L } \\
(n=31)\end{array}$ & $\begin{array}{l}\mathrm{p}- \\
\text { value }\end{array}$ \\
\hline Age (years) ${ }^{a}$ & $\begin{array}{l}56.6 \pm \\
2.4\end{array}$ & $\begin{array}{l}55.6 \pm \\
3.4\end{array}$ & $\begin{array}{l}56.3 \pm \\
2.6\end{array}$ & n.s. \\
\hline Sex (male/ female) ${ }^{b}$ & $8 / 23$ & $14 / 48$ & $9 / 22$ & n.s. \\
\hline Affected side (right/ left) ${ }^{b}$ & $16 / 15$ & $35 / 27$ & $16 / 15$ & n.s. \\
\hline Body mass index $\left(\mathrm{kg} / \mathrm{m}^{2}\right)^{a}$ & $\begin{array}{l}26.5 \pm \\
2.5\end{array}$ & $\begin{array}{l}26.3 \pm \\
2.6\end{array}$ & $\begin{array}{l}26.8 \pm \\
2.7\end{array}$ & n.s. \\
\hline Follow-up period (months) ${ }^{a}$ & $\begin{array}{l}38.2 \pm \\
3.6\end{array}$ & $\begin{array}{l}38.9 \pm \\
4.7\end{array}$ & $\begin{array}{l}39.1 \pm \\
5.5\end{array}$ & n.s. \\
\hline $\begin{array}{l}\text { Time interval from index surgery to removal surgery } \\
\text { (months) }{ }^{a}\end{array}$ & $\begin{array}{l}24.7 \pm \\
3.0\end{array}$ & $\begin{array}{l}24.8 \pm \\
3.1\end{array}$ & $\begin{array}{l}24.9 \pm \\
2.7\end{array}$ & n.s. \\
\hline \multicolumn{5}{|l|}{ a The values are given as mean \pm standard deviation } \\
\hline${ }^{\mathrm{b}}$ The values are given as a $\mathrm{n}(\%)$ & & & & \\
\hline
\end{tabular}


Table 2

Preoperative clinical, radiological, and arthroscopic evaluations

\begin{tabular}{|c|c|c|c|c|}
\hline Variable & $\begin{array}{l}\text { Group S } \\
(n=31)\end{array}$ & $\begin{array}{l}\text { Group M } \\
(n=62)\end{array}$ & $\begin{array}{l}\text { Group L } \\
(n=31)\end{array}$ & p-value \\
\hline Lysholm knee score ${ }^{a}$ & $53.8 \pm 12.2$ & $53.4 \pm 9.7$ & $51.9 \pm 10.6$ & n.s. \\
\hline KOOS Pain ${ }^{a}$ & $55.5 \pm 8.0$ & $56.4 \pm 8.5$ & $54.8 \pm 8.2$ & n.s. \\
\hline KOOS Symptoms $^{\text {a }}$ & $54.7 \pm 8.7$ & $56.2 \pm 8.9$ & $53.9 \pm 9.0$ & n.s. \\
\hline KOOS Activities of Daily Living ${ }^{a}$ & $58.4 \pm 7.7$ & $58.1 \pm 7.9$ & $57.7 \pm 8.0$ & n.s. \\
\hline KOOS Sports / Rec ${ }^{a}$ & $34.5 \pm 12.8$ & $34.1 \pm 12.5$ & $33.7 \pm 12.4$ & n.s. \\
\hline $\begin{array}{l}\text { KOOS Quality of Life }{ }^{\text {a }} \\
\text { (knee-related) }\end{array}$ & $35.7 \pm 9.0$ & $36.1 \pm 9.2$ & $35.1 \pm 9.5$ & n.s. \\
\hline Shelbourne and Trumper score ${ }^{a}$ & $94.8 \pm 3.3$ & $94.5 \pm 5.6$ & $94.2 \pm 5.5$ & n.s. \\
\hline \multicolumn{4}{|l|}{ IKDC radiographic scale ${ }^{b}$} & n.s. \\
\hline 1 & $18(58.1 \%)$ & $36(58.1 \%)$ & $18(58.1 \%)$ & \\
\hline 2 & $12(38.7 \%)$ & $23(37.1 \%)$ & $13(41.9 \%)$ & \\
\hline 3 & $1(3.2 \%)$ & $3(4.8 \%)$ & $0(0 \%)$ & \\
\hline Hip-knee-ankle angle $\left(^{\circ}\right)^{a}$ & $-6.9 \pm 2.5$ & $-8.2 \pm 2.1$ & $-10.0 \pm 3.4$ & $<0.001$ \\
\hline Modified Blackburn-Peel ratio a & $0.9 \pm 0.1$ & $0.9 \pm 0.1$ & $0.9 \pm 0.2$ & n.s \\
\hline \multicolumn{5}{|c|}{ KOOS: Knee Injury and Osteoarthritis Outcome Score; } \\
\hline \multicolumn{5}{|c|}{ IKDC: International Knee Documentation Committee } \\
\hline a The values are given as mean \pm stan & Jard deviation & & & \\
\hline
\end{tabular}

Comparison of postoperative outcomes showed statistical significant differences in terms of Shelbourne and Trumper score: Group S = 89.8 \pm 7.1 , Group $M=89.3 \pm 7.6$, and Group L = 84.8 $\pm 5.7(p=0.008)$ (Table 3). Subsequent post-hoc multiple comparison analysis revealed significant differences between Group $L$ and the other two groups, while no significance was evident between Groups S and M (Table 4). Other postoperative functional evluations such as Lysholm knee score and KOOS subscales did not show statistically significant. Postoperative comparisons of IKDC radiographic scales, hip-knee-ankle angle, and modified Blackburn-Peel ratio had no statistical significant difference between the groups (Table 3). The interobserver and intraobserver reliabilities measured preoperatively were 0.811 and 0.883 for MPTA, 
0.872 and 0.897 for hip-knee-ankle angle, and 0.802 and 0.839 for modified Blackburn-Peel ratio, and 0.782 and 0.853 for IKDC radiographic scale, while the postoperative interobserver and intraobserver reliabilities for radiographic parameters including the MPTA (0.791 and 0.871), hip-knee-ankle angle (0.835 and 0.911), modified Blackburn-Peel ratio (0.772 and 0.829), and IKDC radiographic scale (0.759 and 0.868$)$, respectively.

Table 3

Postoperative clinical, and radiologic evaluations

\begin{tabular}{|c|c|c|c|c|}
\hline Variable & $\begin{array}{l}\text { Group S } \\
(n=31)\end{array}$ & $\begin{array}{l}\text { Group M } \\
(n=62)\end{array}$ & $\begin{array}{l}\text { Group L } \\
(n=31)\end{array}$ & p-value \\
\hline Lysholm knee score $^{a}$ & $73.4 \pm 7.6$ & $74.4 \pm 9.3$ & $72.9 \pm 8.8$ & n.s. \\
\hline Koos Pain ${ }^{a}$ & $68.3 \pm 7.3$ & $68.9 \pm 8.1$ & $67.4 \pm 7.5$ & n.s. \\
\hline KOOS Symptoms $^{a}$ & $67.4 \pm 7.0$ & $67.7 \pm 6.8$ & $66.0 \pm 6.8$ & n.s. \\
\hline KOOS Activities of Daily Living a & $69.3 \pm 7.1$ & $68.7 \pm 7.1$ & $68.0 \pm 7.1$ & n.s. \\
\hline KOOS Sports/Rec ${ }^{a}$ & $50.7 \pm 9.6$ & $51.1 \pm 9.9$ & $48.9 \pm 9.5$ & n.s. \\
\hline $\begin{array}{l}\text { KOOS Quality of Life a } \\
\text { (knee-related) }\end{array}$ & $59.1 \pm 8.7$ & $59.2 \pm 8.7$ & $57.5 \pm 7.8$ & n.s. \\
\hline Shelbourne and Trumper score ${ }^{a}$ & $89.8 \pm 7.1$ & $89.3 \pm 7.6$ & $84.8 \pm 5.7$ & $0.008^{a}$ \\
\hline Bony correction angle $\left({ }^{\circ}\right)^{a}$ & $6.9 \pm 0.6$ & $10.0 \pm 1.1$ & $13.2 \pm 1.0$ & $<0.001^{\mathrm{a}}$ \\
\hline \multicolumn{4}{|l|}{ IKDC radiographic scale ${ }^{b}$} & \multirow[t]{4}{*}{ n.s. } \\
\hline A & $17(54.8 \%)$ & $30(48.4 \%)$ & $14(45.1 \%)$ & \\
\hline B & $12(38.7 \%)$ & $29(46.8 \%)$ & $15(48.4 \%)$ & \\
\hline C & $2(6.5 \%)$ & $3(4.8 \%)$ & $2(6.4 \%)$ & \\
\hline Hip-knee-ankle angle $\left({ }^{\circ}\right)^{\text {a }}$ & $3.4 \pm 1.1$ & $3.6 \pm 1.4$ & $3.7 \pm 1.6$ & n.s. \\
\hline Modified Blackburn-Peel ratio ${ }^{a}$ & $0.8 \pm 0.1$ & $0.7 \pm 0.1$ & $0.7 \pm 0.1$ & n.s. \\
\hline \multicolumn{5}{|c|}{ KOOS: Knee Injury and Osteoarthritis Outcome Score; } \\
\hline \multicolumn{5}{|c|}{ IKDC: International Knee Documentation Committee } \\
\hline \multicolumn{5}{|c|}{${ }^{\text {a }}$ The values are given as a mean \pm standard deviation } \\
\hline${ }^{\mathrm{b}}$ The values are given as a $\mathrm{n}(\%)$ & & & & \\
\hline
\end{tabular}


Table 4

Pairwise comparison of postoperative variables between each group

\begin{tabular}{|llll|}
\hline Variable & $\begin{array}{l}\text { Group S vs. Group } \\
\text { M }\end{array}$ & $\begin{array}{l}\text { Group S vs. Group } \\
\text { L }\end{array}$ & Group M vs. Group L \\
\hline $\begin{array}{l}\text { Shelbourne and Trumper } \\
\text { score }\end{array}$ & n.s. & 0.018 & 0.015 \\
\hline Bonferroni's test was used for post-hoc analysis and is represented as an adjusted p-value. \\
\hline
\end{tabular}

Between first- and second-look arthroscopies, progression rates of cartilage degeneration evident on the femoral trochlea in Groups S, M, and L were $22.6 \%, 40.3 \%$, and $58.1 \%$, respectively ( $p=0.017)$. For cartilage on the patellar surface, Groups $S, M$, and $L$ showed $12.9 \%, 19.4 \%$, and $38.7 \%$ of cases having progression of degeneration, respectively $(p=0.036)$ (Supplement 1$)$. Proportion of progression of cartilage degeneration found on both surfaces of the femoral trochlea and patella differ significantly between the groups. The statistical power was $72.4 \%$ with regard to progression in cartilage status of the femoral trochlea and $63.1 \%$ with regard to progression in cartilage status of the patella. Result of the regression demonstrated that only the correction angle affected significantly on progression of the cartilage status of both the patella and femoral trochlea joint (Table 5). 
Table 5

Logistic regression analysis of association between independent variables and progression of the ICRS grade

\begin{tabular}{|lll|}
\hline & OR $(95 \% \mathrm{Cl})$ & p-value \\
\hline Femoral trochlea progression & & \\
\hline Age & $1.023(0.906,1.155)$ & n.s. \\
\hline Sex & $1.949(0.693,5.514)$ & n.s. \\
\hline Body mass index & $1.016(0.884,1.168)$ & n.s. \\
\hline Preoperative hip-knee-ankle angle & $1.041(0.914,1.185)$ & n.s. \\
\hline Postoperative modified Blackburn-Peel ratio & $0.42(0.012,14.968)$ & n.s. \\
\hline Bony correction angle & $1.233(1.054,1.443)$ & 0.009 \\
\hline Patella progression & & \\
\hline Age & $0.989(0.859,1.139)$ & n.s. \\
\hline Sex & $1.709(0.588,4.965)$ & n.s. \\
\hline Body mass index & $0.907(0.763,1.077)$ & n.s. \\
\hline Preoperative hip-knee-ankle angle & $1.030(0.887,1.197)$ & n.s. \\
\hline Postoperative modified Blackburn-Peel ratio & $0.270(0.004,17.264)$ & n.s. \\
\hline Bony correction angle & $1.216(1.015,1.458)$ & 0.034 \\
\hline OR: odds ratio; Cl: confidence interval & & \\
\hline Figure 1 & & \\
\hline
\end{tabular}

\section{Discussion}

The purpose of this study was to investigate the relation of bony correction angles achieved after MOWHTO to the state of patellofemoral joint cartilage and clinical outcomes. In this study, we discovered that (1) there was cartilage lesion progression in $40.3 \%$ of the femoral trochleas and $22.6 \%$ of the patellas included in this study; (2) the cartilages were more significantly deteriorated when the bony correction angle to be achieved became larger; and (3) there was no significant difference evident in most clinical outcomes measured, including Lysholm score and the KOOS subscales, with the exception of postoperative ST score.

The primary finding of the current study was that there is a correlation between MOWHTO and postoperative arthritic progression in the patellofemoral joint. Agneskirchner et al.[12] noted in their biomechanical cadaveric study, which investigated changes in the patellofemoral joint after the 
osteotomy, that lateralization and distalization of the tibial tuberosity produces loss in patellar height. This leads to elevation in patellofemoral contact pressure and, in turn, the loss results in more incidence of anterior knee pain with a progression of cartilage degeneration in the joint. Based on their biomechanical study, Goshima et al.[17] and Kim et al.[18] further looked into (or assessed/investigated) the clinical aspects of the articular cartilage and their findings confirmed progressive degeneration through second arthroscopy performed in patients who had undergone MOWHTO previously. Our study found that about $41.1 \%$ of all patients had progression, and we consider this high prevalent rate of cartilage degeneration in the joint to be a direct result of patellofemoral pressure elevation subsequent to the surgery.

We also demonstrated that Group $L$ with a higher bony correction angle showed significantly more extensive cartilage progression than did the other two groups with less correction. Each articular cartilage on both the patella and femoral trochlea revealed significant differences in progression among the groups [Group $S=22.6 \%$, Group $M=40.3 \%$, and Group $L=58.1 \%$ for the femoral trochlea $(p=0.017)$ and Group $S=12.9 \%$, Group $M=19.4 \%$, and Group $L=38.7 \%$ for the patella $(p=0.036)]$. Following logistic regression, we also discovered statistical differences in the angle of correction. Javidan et al.,[14] through a biomechanical study, noticed that MOWHTO leads to a rise in the patellofemoral joint's contact pressure and also that there was a significantly greater increase of contact pressure in $15 \mathrm{~mm}$ widening than in $10 \mathrm{~mm}$ widening. Our clinical results were comparable to their study's outcomes. The reason for higher prevalence of cartilage degeneration in the group with a larger correction angle is in accordance with the results of the biomechanical studies, in which it was found that a larger correction angle consequently further promotes distalization and lateralization of the patella, resulting in a much higher contact pressure of the patellofemoral joint, as found in Javidan et al.'s study.

Goshima et al.[17] performed second-look arthroscopy in 60 cases at 19 months after the initial MOWHTO procedure on average and found that $45 \%$ of the patients showed progressive degeneration of the cartilage with respect to ICRS grade. However, when the mean follow-up time was extended to 58.2 months, there was no definite correlation of this degeneration with clinical outcomes. There may not be much clinical effect of loss in patellar height on significantly undesirable patellofemoral symptoms as they mentioned, but we believe that a larger correction angle would cause a significantly higher loss in the height, resulting in a rather dissimilar outcome versus their findings. In addition, their score system for evaluating outcomes was more oriented to general knee symptoms rather than focusing on the patellofemoral joint, and only some of their scoring items were related with this particular joint. Thus, it can be postulated that, if their patients' main symptoms had been originating from the medial compartment, then the procedure performed considerably relieved these symptoms while discriminating developing symptoms related to the patellofemoral joint wherein postoperative anatomical alteration is relatively small compared to a change in coronal axis alignment of the medial compartment. To avoid this discrimination, our study used ST score to more closely evaluate anterior knee pain. Our results showed that postoperative ST score was lower than the score measured preoperatively in all groups, and, in particular, the score was significantly lower in Group $L$ than in the other two groups. Thus, considering these findings, careful patient selection is imperative, because, in patients who are expected to require a 
large correction angle during MOWHTO, patellofemoral joint symptoms may be incited and aggravated within a relatively short duration after the surgery.

\section{Limitations}

The present study had several limitations. First, there might be a selection bias in our study because it is a retrospective study based on medical records, instead of being a prospective controlled study. However, we conclude that our result is still reliable since there was no difference in preoperative demographic, clinical and radiological data among three groups. Second, the duration from MOWHTO to second look arthroscopy was 24.8 months, which was not enough to conduct long term research including cartilage status and degenerative change. However, any articular changes that occur are evident earlier under arthroscopy than are the changes that would be visible on radiography, and our utilization of second-look arthroscopy to detect any early changes and the application of these findings in our analysis would help to compensate for our relatively short follow-up time. Lastly, no control group with comparable demographic characteristics who did not undergo MOWHTO was available to make a direct comparison of our findings to the effects of normal age-dependent joint degeneration, since there is a possibility that age-dependent joint degeneration may be a culprit in our finding of patellofemoral joint's cartilage degeneration. This possibility should be evaluated using the same two-stage arthroscopy protocol without undergoing the correction surgery over a period of several years. However, it is considered unethical and impractical to perform multiple arthroscopies without MOWHTO in patients with known medial compartment osteoarthritis of their knee joint merely to examine changes in the patellofemoral joint.

\section{Conclusion}

When the correction angle to be achieved during MOWHTO becomes larger, such leads to more prevalent and substantial cartilage degeneration in the patellofemoral joint with further adverse clinical outcomes. Thus, in cases that require considerable bony angle correction, careful patient selection is necessary since these patients may suffer from subsequent patellofemoral joint symptoms within a relatively short time after the procedure.

\section{References}

1. Debeyre J: [Surgical treatment of arthroses of the knee]. Sem Hop 1951, 27(61-62):2515-2518.

2. Akizuki S, Shibakawa A, Takizawa T, Yamazaki I, Horiuchi H: The long-term outcome of high tibial osteotomy: a ten- to 20-year follow-up. J Bone Joint Surg Br 2008, 90(5):592-596.

3. Floerkemeier S, Staubli AE, Schroeter S, Goldhahn S, Lobenhoffer P: Outcome after high tibial openwedge osteotomy: a retrospective evaluation of $\mathbf{5 3 3}$ patients. Knee Surg Sports Traumatol Arthrosc 2013, 21(1):170-180. 
4. Schallberger A, Jacobi M, Wahl P, Maestretti G, Jakob RP: High tibial valgus osteotomy in unicompartmental medial osteoarthritis of the knee: a retrospective follow-up study over 13-21 years. Knee Surg Sports Traumatol Arthrosc 2011, 19(1):122-127.

5. Schuster P, Schulz M, Mayer P, Schlumberger M, Immendoerfer M, Richter J: Open-Wedge High Tibial Osteotomy and Combined Abrasion/Microfracture in Severe Medial Osteoarthritis and Varus Malalignment: 5-Year Results and Arthroscopic Findings After 2 Years. Arthroscopy 2015, 31(7):1279-1288.

6. Conventry MB: Osteotomy of the upper portion of the tibia for degenerative arthritis of the knee. A preliminary report by Mark B. Conventry, MD. From the Section of Orthopedic Surgery, Mayo Clinic and Mayo Foundation, Rochester, Minnesota. 1965. J Bone Joint Surg Am 2001, 83-a(9):1426.

7. Lobenhoffer P, Agneskirchner JD: Improvements in surgical technique of valgus high tibial osteotomy. Knee Surg Sports Traumatol Arthrosc 2003, 11(3):132-138.

8. Sterett WI, Steadman JR, Huang MJ, Matheny LM, Briggs KK: Chondral resurfacing and high tibial osteotomy in the varus knee: survivorship analysis. Am J Sports Med 2010, 38(7):1420-1424.

9. Jung WH, Takeuchi R, Chun CW, Lee JS, Ha JH, Kim JH, Jeong JH: Second-look arthroscopic assessment of cartilage regeneration after medial opening-wedge high tibial osteotomy. Arthroscopy 2014, 30(1):72-79.

10. Kanamiya $T$, Naito $M$, Hara $M$, Yoshimura I: The influences of biomechanical factors on cartilage regeneration after high tibial osteotomy for knees with medial compartment osteoarthritis: clinical and arthroscopic observations. Arthroscopy 2002, 18(7):725-729.

11. Kim CW, Seo SS, Lee CR, Gwak HC, Kim JH, Jung SG: Factors affecting articular cartilage repair after open-wedge high tibial osteotomy. Knee 2017, 24(5):1099-1107.

12. Agneskirchner JD, Hurschler C, Wrann CD, Lobenhoffer P: The effects of valgus medial opening wedge high tibial osteotomy on articular cartilage pressure of the knee: a biomechanical study. Arthroscopy 2007, 23(8):852-861.

13. Gaasbeek R, Welsing R, Barink M, Verdonschot N, van Kampen A: The influence of open and closed high tibial osteotomy on dynamic patellar tracking: a biomechanical study. Knee Surg Sports Traumatol Arthrosc 2007, 15(8):978-984.

14. Javidan P, Adamson GJ, Miller JR, Durand P, Jr., Dawson PA, Pink MM, Lee TQ: The effect of medial opening wedge proximal tibial osteotomy on patellofemoral contact. Am J Sports Med 2013, 41(1):80-86.

15. Stoffel K, Willers C, Korshid O, Kuster M: Patellofemoral contact pressure following high tibial osteotomy: a cadaveric study. Knee Surg Sports Traumatol Arthrosc 2007, 15(9):1094-1100.

16. Kaper BP, Bourne RB, Rorabeck CH, Macdonald SJ: Patellar infera after high tibial osteotomy. $J$ Arthroplasty 2001, 16(2):168-173.

17. Goshima K, Sawaguchi T, Shigemoto K, Iwai S, Nakanishi A, Ueoka K: Patellofemoral Osteoarthritis Progression and Alignment Changes after Open-Wedge High Tibial Osteotomy Do Not Affect Clinical Outcomes at Mid-term Follow-up. Arthroscopy 2017, 33(10):1832-1839. 
18. Kim KI, Kim DK, Song SJ, Lee SH, Bae DK: Medial Open-Wedge High Tibial Osteotomy May Adversely Affect the Patellofemoral Joint. Arthroscopy 2017, 33(4):811-816.

19. Lee YS, Lee SB, Oh WS, Kwon YE, Lee BK: Changes in patellofemoral alignment do not cause clinical impact after open-wedge high tibial osteotomy. Knee Surg Sports Traumatol Arthrosc 2016, 24(1):129-133.

20. Outerbridge RE: The etiology of chondromalacia patellae. J Bone Joint Surg Br 1961, 43-b:752-757.

21. Ogawa H, Matsumoto K, Ogawa T, Takeuchi K, Akiyama H: Preoperative varus laxity correlates with overcorrection in medial opening wedge high tibial osteotomy. Arch Orthop Trauma Surg 2016, 136(10):1337-1342.

22. Miniaci A, Ballmer FT, Ballmer PM, Jakob RP: Proximal tibial osteotomy. A new fixation device. Clin Orthop Relat Res 1989(246):250-259.

23. Fujisawa $\mathrm{Y}$, Masuhara $\mathrm{K}$, Shiomi S: The effect of high tibial osteotomy on osteoarthritis of the knee. An arthroscopic study of 54 knee joints. Orthop Clin North Am 1979, 10(3):585-608.

24. Song EK, Seon JK, Park SJ: How to avoid unintended increase of posterior slope in navigationassisted open-wedge high tibial osteotomy. Orthopedics 2007, 30(10 Suppl):S127-131.

25. Hefti F, Muller W, Jakob RP, Staubli HU: Evaluation of knee ligament injuries with the IKDC form. Knee Surg Sports Traumatol Arthrosc 1993, 1(3-4):226-234.

26. Lysholm J, Gillquist J: Evaluation of knee ligament surgery results with special emphasis on use of a scoring scale. Am J Sports Med 1982, 10(3):150-154.

27. Roos EM, Roos HP, Lohmander LS, Ekdahl C, Beynnon BD: Knee Injury and Osteoarthritis Outcome Score (KOOS)-development of a self-administered outcome measure. J Orthop Sports Phys Ther 1998, 28(2):88-96.

28. Shelbourne KD, Trumper RV: Preventing anterior knee pain after anterior cruciate ligament reconstruction. Am J Sports Med 1997, 25(1):41-47.

\section{Declarations}

Acknowledgements

Not applicable

Funding

Each author certifies that he or she has no commercial associations (eg, consultancies, stock ownership, equity interest, patent/licensing arrangements, etc) that might pose a conflict of interest in connection with the submitted article. There were no external sources of funding and none of the authors had any conflicts of interest.

Ethics declarations 


\section{Ethics and consent to participate}

This study was ethically approved by the Institutional Review Board (IRB) of Severance hospital (ID Number: 4-2017-0737). Consent was not required by the institution ethical committee for this retrospective study.

\section{Consent for publication}

Not applicable

\section{Competing interests}

The authors declare that they have no competing interests.

\section{Author's contribution}

The project was coordinated by TH Yoon, $\mathrm{CH}$ Choi, and J Min. TH Yoon drafted the manuscript, together with M Jung, SH Kim, and CH Choi. SH Kim generated the concept of the study. The design of the study, acquisition of data, analysis and interpretation of data were all done jointly by all authors. TH Yoon, and SH Kim revised the final draft critically for important intellectual content and approved the version to be submitted. All of authors agreed to be accountable for all aspects of the work in ensuring that questions related to the accuracy or integrity of any part of the work are appropriately investigated and resolved.

\section{Availability of data and materials}

The datasets used and/or analyzed in this study available from the corresponding author on reasonable request.

\section{Figures}




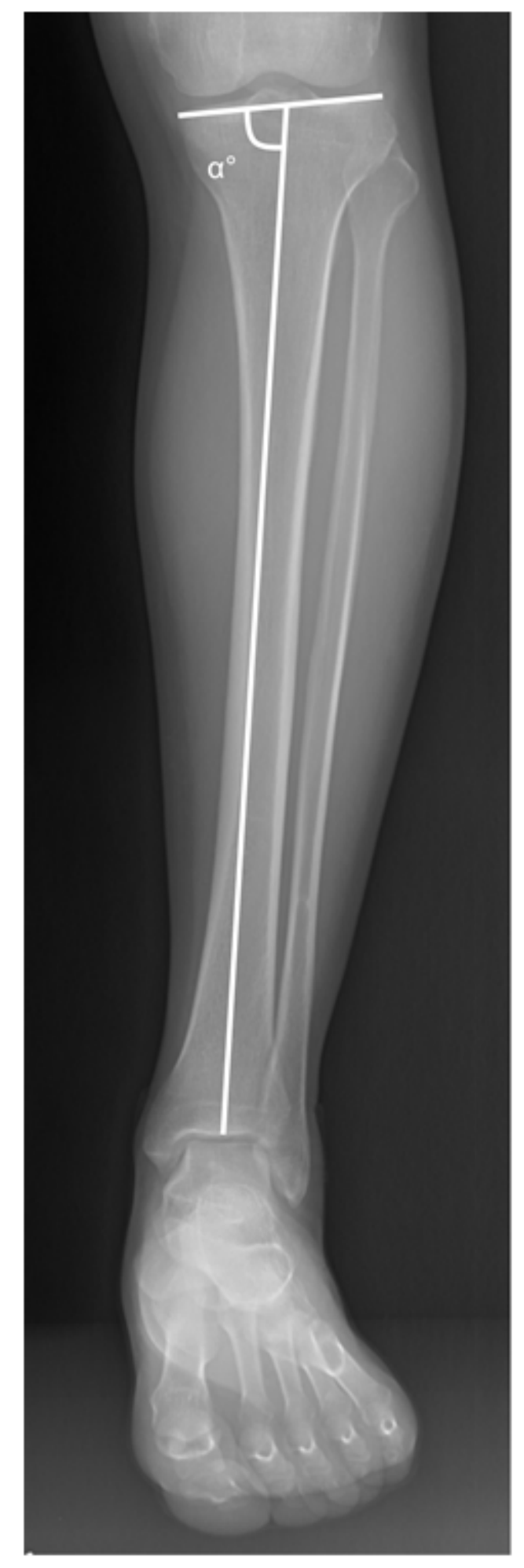

\section{Figure 1}

The medial proximal tibial angle was defined as the angle obtained from an intersection between the articular surface of the proximal tibia and the tibial mechanical axis. $\left(a^{\circ}\right)$ 


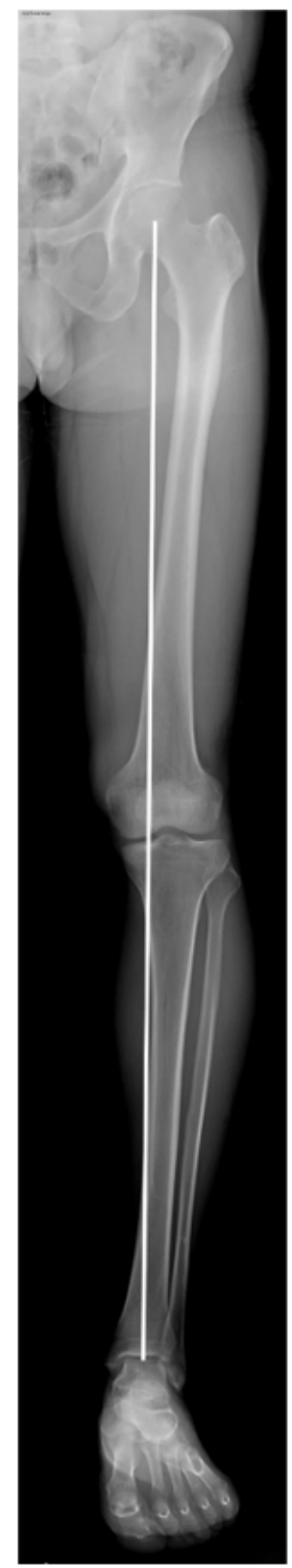

\section{Figure 2}

Radiologic measurements. (A) The weight bearing line is drawn from the center of the femoral head to the midpoint of the superior articular surface of the talus. (B) The weight bearing line ratio is calculated as the ratio of the horizontal distance from the medial edge of the tibia plateau to the intersection of the weight bearing line and the proximal tibia plateau line to the entire length of the proximal tibia plateau line. $(\mathrm{a} / \mathrm{b})(\mathrm{C})$ The hip-knee-ankle angle was obtained by drawing a line from the center of the femoral 
head to the midpoint of the tibial spine along with second line extended from this midpoint to the center of the talar surface of the ankle joint. $\left(\beta^{\circ}\right)(D)$ The modified Blackburne-Peel ratio is defined as the perpendicular height from the tibial articular surface to the inferior aspect of the patellar articular surface divided by the length of the patellar articular surface (c/d).

Figure 3(A)

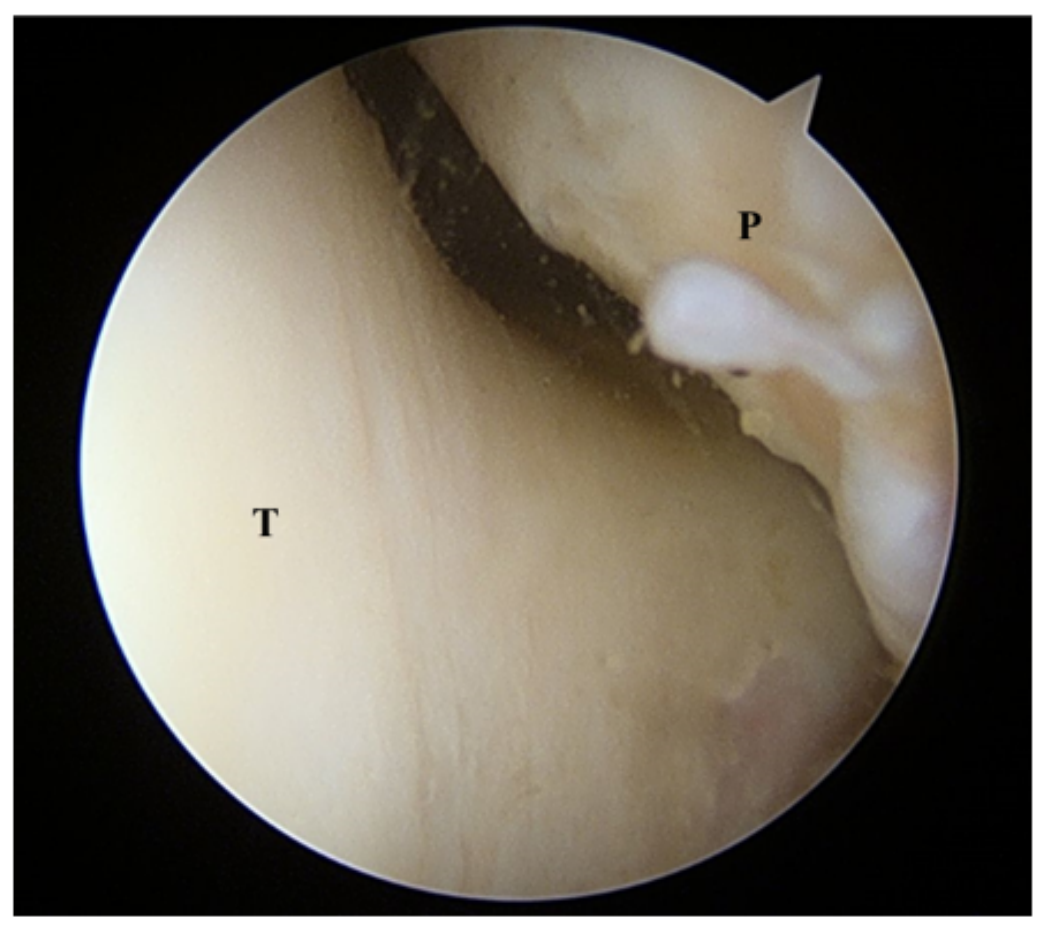

Figure 3(B)

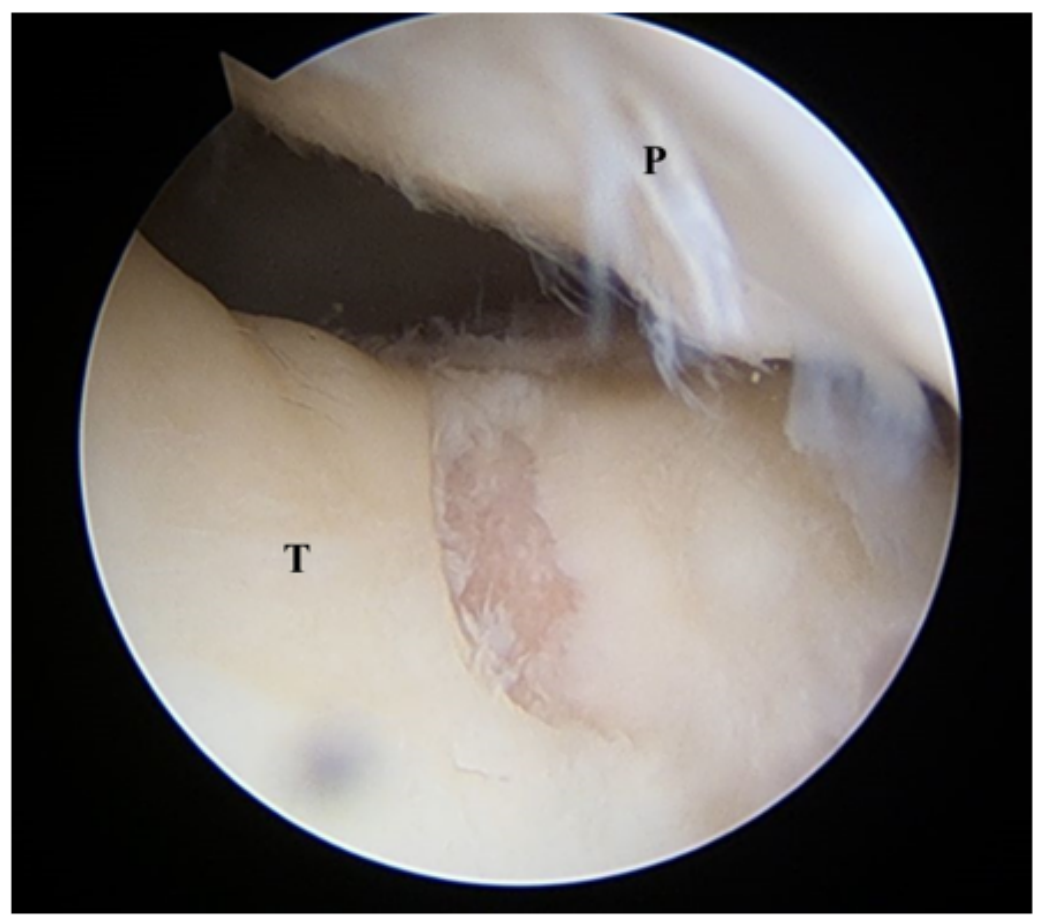

Figure 3 
Arthroscopic findings of the right knee in a 61-year-old female. $T=$ femoral trochlea and $P=$ patella. (A) The first arthroscopy during the osteotomy showed cartilage lesions of the ICRS grade I on the femoral trochlea. (B) The second-look arthroscopy during implant removal performed at 24.5 months postoperatively showed progression of the previously noted cartilage lesions (ICRS grade IV on the femoral trochlea).

\section{Supplementary Files}

This is a list of supplementary files associated with this preprint. Click to download.

- Supplement1.docx 